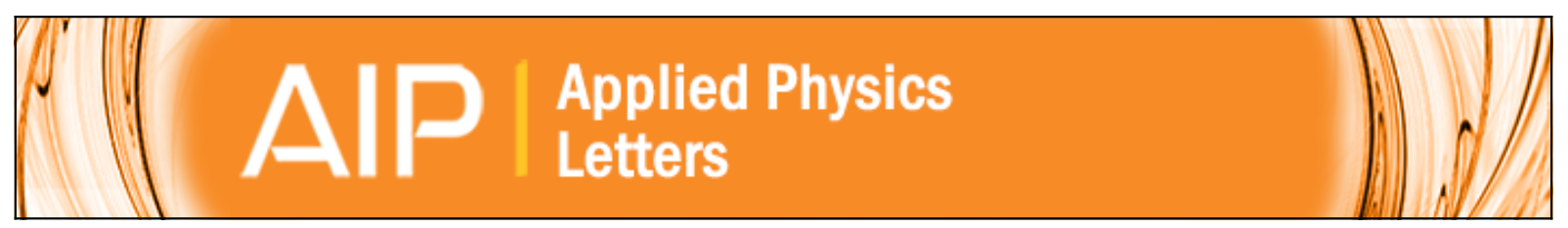

Identification of screw dislocations as fast-forming sites in Fe-doped $\mathrm{SrTiO}_{3}$

Ch. Lenser, Z. Connell, A. Kovács, R. Dunin-Borkowski, A. Köhl, R. Waser, and R. Dittmann

Citation: Applied Physics Letters 102, 183504 (2013); doi: 10.1063/1.4804364

View online: http://dx.doi.org/10.1063/1.4804364

View Table of Contents: http://scitation.aip.org/content/aip/journal/apl/102/18?ver=pdfcov

Published by the AIP Publishing 


\title{
Identification of screw dislocations as fast-forming sites in Fe-doped $\mathrm{SrTiO}_{3}$
}

\author{
Ch. Lenser, ${ }^{1, a)}$ Z. Connell, ${ }^{2}$ A. Kovács, ${ }^{1,3}$ R. Dunin-Borkowski, ${ }^{1,3}$ A. Köhl, ${ }^{1}$ R. Waser,,${ }^{1,4}$ \\ and R. Dittmann ${ }^{1}$ \\ ${ }^{1}$ Peter Grünberg Institute, Forschungszentrum Jülich, 52425 Jülich, Germany \\ ${ }^{2}$ Department of Materials Science and Engineering and Materials Research Laboratory, University of Illinois, \\ Urbana-Champaign, Urbana, Illinois 61801, USA \\ ${ }^{3}$ Ernst Ruska-Centre for Microscopy and Spectroscopy with Electrons, Forschungszentrum Jülich, \\ 52425 Jülich, Germany \\ ${ }^{4}$ Institute of Materials in Electrical Engineering and Information Technology II, RWTH Aachen University, \\ 52056 Aachen, Germany
}

(Received 29 January 2013; accepted 21 April 2013; published online 7 May 2013)

\begin{abstract}
The defect-mediated nature of resistance switching in transition-metal oxides necessitates a detailed understanding of the defect structure and its influence on the electrical properties of the material. We show that screw dislocations in the substrate induce growth defects that strongly influence the electrical properties of metal-insulator-metal structures fabricated from epitaxial Fe-doped $\mathrm{SrTiO}_{3}$ (STO) thin films. Direct comparison of Pt/Fe:STO/Nb:STO cells with and without growth defects shows a reduction of the electro-forming time of more than three orders of magnitude when a defect is present. We propose an explanation centered on the physical properties of grain boundaries in acceptor-doped $\mathrm{SrTiO}_{3}$. C 2013 AIP Publishing LLC. [http://dx.doi.org/10.1063/1.4804364]
\end{abstract}

Resistively switchable oxides have attracted great scientific attention over the past 10 years due to the potential application of such materials in future non-volatile memory technology. ${ }^{1,2}$ The changes in the resistive state of resistive random access memory (ReRAM) devices are generally attributed to the movement of oxygen vacancies-which have donor-character in oxides-during electrical biasing, and the resulting modification of the dopant profiles in such devices. $^{3,4}$ Most oxides that are being investigated for ReRAM applications (such as $\mathrm{TiO}_{2}, \mathrm{SrTiO}_{3}(\mathrm{STO}), \mathrm{HfO}_{2}$, $\mathrm{TaO}_{2}$ ) are highly insulating in the electrically untreated state, and the high resistance has to be broken down by a dedicated DC voltage treatment. ${ }^{4-7}$ This electroforming process is generally understood as a removal of lattice oxygen and an accumulation of point defects, namely, oxygen vacancies, along a filamentary pathway that forms the conducting filament. ${ }^{1,8}$ Once the forming is complete, the redox-state of the conductive filament can be reversibly changed close to the top electrode by bipolar voltage treatment. A complementary approach uses reactive electrodes to substitute the electrical forming by a chemical reduction that alters the electrical properties of the insulator. ${ }^{9}$

The importance of defects for the resistive switching phenomenon has been widely recognized, and numerous studies focus on point- and extended defects and their movement and stability in the oxide lattice. ${ }^{1,2,10}$ Detailed studies performed on dislocations in $\mathrm{SrTiO}_{3}$ have shown that dislocation cores have much better conductivity than the lattice, which can be attributed to oxygen vacancies along the dislocation core. ${ }^{11,12}$ In addition to crystalline defects, Rossel et al. point out the impact of microstructural defects on the current distribution in metal-insulator-metal (MIM) structures and suggest a key role for resistive switching. ${ }^{13}$

In this letter, we demonstrate the importance of substrate-induced defects for the electrical properties of

a)c.lenser@fz-juelich.de
MIM structures for the specific case of screw-dislocations, which are intrinsic defects to every crystalline material. The investigated $\mathrm{SrTi}_{1-x} \mathrm{Fe}_{x} \mathrm{O}_{3}(\mathrm{x}=0.02,0.05)$ films were grown epitaxially onto commercially available $\mathrm{Nb}$-doped $\mathrm{SrTiO}_{3}$ substrates (Crystec, Berlin, Germany) by pulsed laser deposition (PLD), at a substrate temperature of $700{ }^{\circ} \mathrm{C}$ and an oxygen pressure $\mathrm{pO}_{2}$ of 0.25 mbar. Prior to film deposition, the as-received crystals were annealed in air for $4 \mathrm{~h}$ at $950{ }^{\circ} \mathrm{C}$ to reconstruct the polished surface. The resulting stepped surface was imaged with atomic force microscopy (AFM) using a Surface Analysis Systems Picostation AFM in tapping mode.

Figure 1(a) shows an exemplary AFM scan taken on the reconstructed surface of a $\mathrm{Nb}$-doped STO single crystal after annealing in air. The surface steps are clearly resolved, and a screw dislocation exit is visible in the center of the image. Due to the favorable half-crystal position surrounding the screw dislocation exit on the surface, diffusing surface atoms preferentially nucleate at the step in the surface. In this manner, screw dislocation exits accumulate additional material during annealing - in the enlarged image in Figure 1(b), the characteristic stepped-pyramidal structure is visible, which is $\approx 9 \mathrm{~nm}$ high and $\approx 700 \mathrm{~nm}$ in diameter.

Figure 1(c) shows a similar crystal after annealing, with a larger surface structure visible in the upper left corner. The zoom in Figure 1(d) reveals that the structure consists of multiple substructures, each of which in turn shows the stepped-pyramidal structure that is characteristic of screwdislocation exits in the crystal surface. These larger structures indicate a possible agglomeration of several screw dislocations in the substrate.

These structures have a strongly increased local density of step-sites, which are preferred nucleation sites for impinging atoms during thin film growth due to the increased number of next-neighbor atoms. As a consequence, the growth rate of the film is strongly increased on a local scale as the growth species diffusing over the surface are trapped at the 

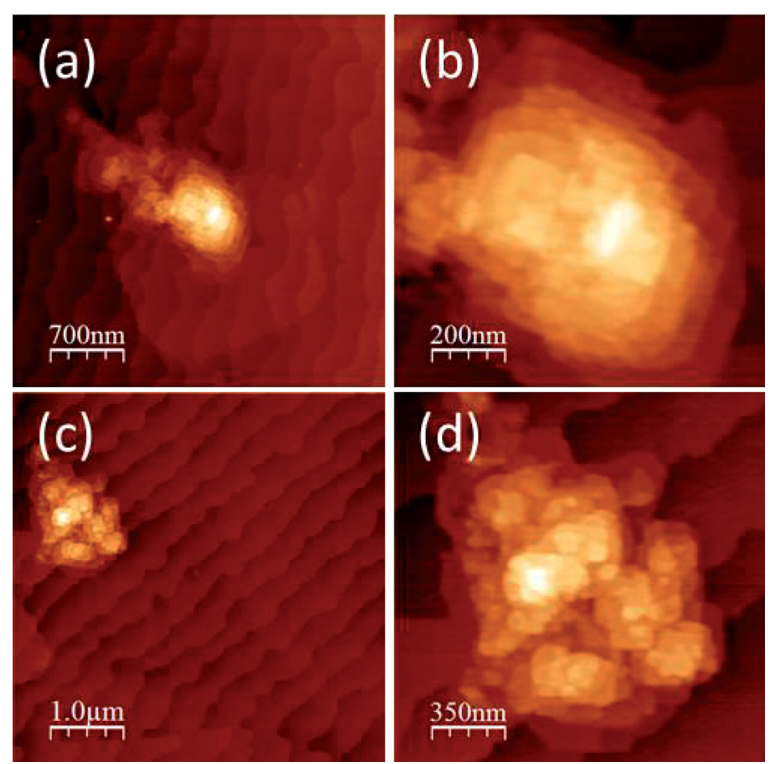

FIG. 1. (a) A single screw dislocation exit on the (001) surface of a Nb:STO crystal after annealing (enlarged in (b)). The color scale runs from 0 to $12 \mathrm{~nm}$ (dark to light). (c) Agglomerated screw dislocations in a similar crystal. The enlarged image in (d) shows several substructures. The color scale runs from 0 to $8 \mathrm{~nm}$ (dark to light).

screw dislocation exit, and specific defect structures can be observed on the surface of the film. Figure 2(a) shows such a surface defect on an as-grown $\mathrm{SrTi}_{0.95} \mathrm{Fe}_{0.05} \mathrm{O}_{3}$ film of $100 \mathrm{~nm}$ thickness, recorded by scanning electron microscopy (SEM) performed in a Hitachi SU 8000 microscope. The diameter of this defect is $\approx 250 \mathrm{~nm}$, and it is made up of inter-

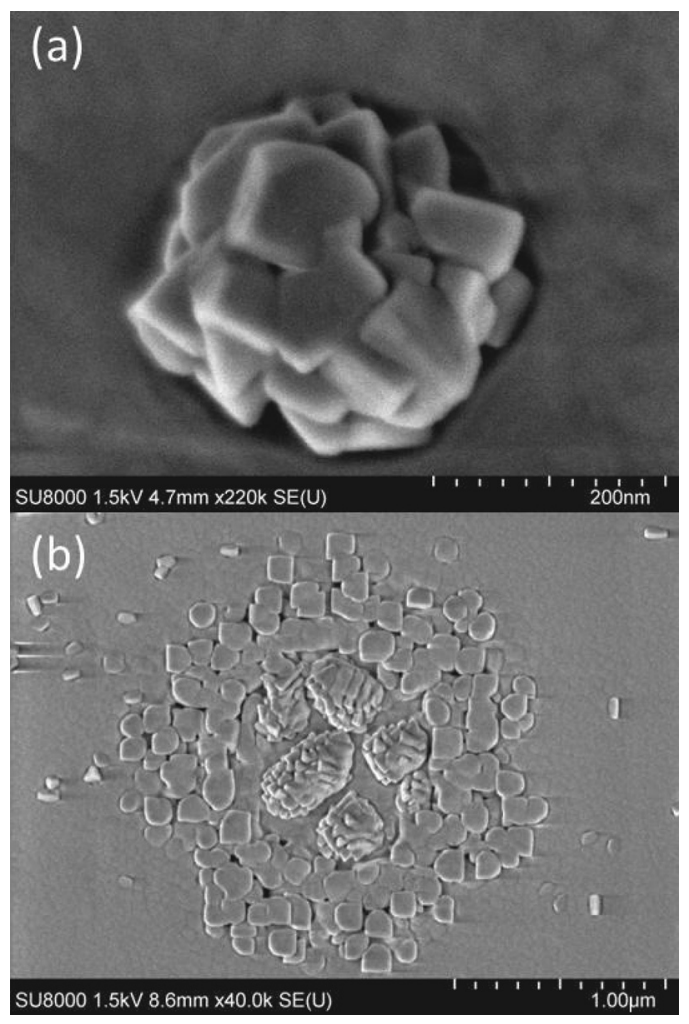

FIG. 2. (a): SEM image of a growth defect on the surface of a $100 \mathrm{~nm}$ $\mathrm{SrTi}_{0.95} \mathrm{Fe}_{0.05} \mathrm{O}_{3}$ film. The cubic habitus of the crystallites indicates polycrystalline STO. (b) SEM image of a much larger surface defect on the same film. The defect consists of several structures, and the epitaxial film around the defect is perturbed. The diameter is approximately $2 \mu \mathrm{m}$. grown STO crystals of cubic habitus. Such defects can be even larger, up to several $\mu \mathrm{m}$ in diameter (see Figure 2(b)). The diameters of these defects are very similar to the size of the regions on the single crystal surface that are perturbed by the screw dislocation exits. One can assume that the diameter of the growth defect is correlated to the size of the perturbation induced by the screw dislocations in the single crystal.

A cross-sectional transmission electron microscopy (TEM) micrograph of a film grown under very similar growth conditions was prepared by focused ion beam (FIB) in a FEI Helios Nanolab 400S dual-beam system. The FIB lamellae were finished using low-energy $\mathrm{Ar}$ ion-milling at $0.5 \mathrm{keV}$ in order to minimize the amount of specimen preparation artifacts. The bright-field TEM image in Figure 3 was recorded using a FEI Tecnai G2 microscope operated at $200 \mathrm{kV}$ and shows a growth defect grown on top of a line defect in the substrate (marked by a white, dashed oval). The growth defect is $\approx 100 \mathrm{~nm}$ high, which is significantly more than the film thickness of this particular epitaxial film $(20 \mathrm{~nm})$. The polycrystalline nature of these defects points to the fact that the growth is too fast to maintain the epitaxial nature of the film, and that nucleation takes place simultaneously at multiple locations of the screw dislocation exit.

To investigate the impact of such growth defects on the electrical properties, a $100 \mathrm{~nm} \mathrm{SrTi}_{0.95} \mathrm{Fe}_{0.05} \mathrm{O}_{3}$ thin film was grown onto a $\mathrm{Nb}: \mathrm{STO}$ single crystal via PLD, and subsequently a Pt marker structure was deposited via optical lithography. Screw dislocation exits were identified via SEM, as shown in Figure 4(a). Next to the large Pt marker visible in the image, a growth defect indicative of a screw dislocation exit can be observed. Pt top electrodes of $10 \times 10 \mu \mathrm{m}^{2}$ were deposited at selected sites via electron beam lithography. Two locations for top electrodes have been indicated in Figure 4(a), one containing a defect (labeled "1") and one on the unperturbed film (labeled "2") for reference. In addition, we confirmed the results presented below on three further electrodes containing a defect and two further reference electrodes without defects.

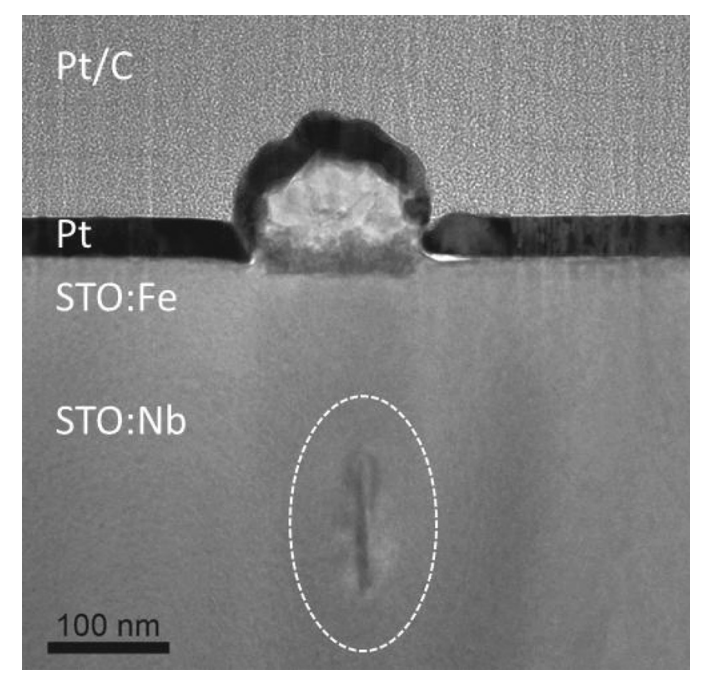

FIG. 3. Brightfield TEM cross-section of a growth defect in a $20 \mathrm{~nm}$ $\mathrm{SrTi}_{0.98} \mathrm{Fe}_{0.02} \mathrm{O}_{3}$ film. The dark line (see white oval) directly underneath the image shows the presence of a line defect in the substrate, which causes the growth defect in the film. The $\mathrm{Pt} / \mathrm{C}$ was deposited as a protective layer during FIB preparation. 

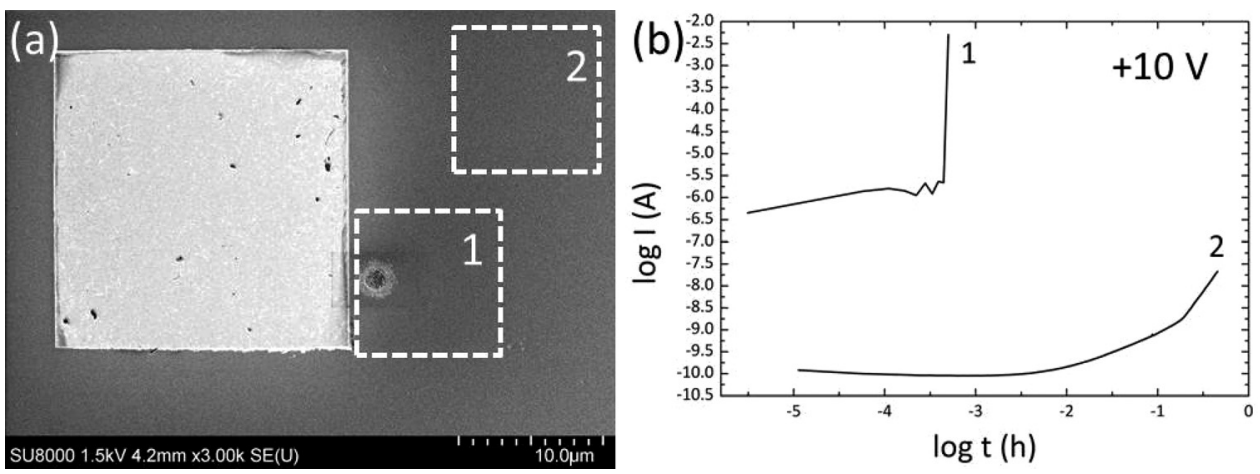

FIG. 4. (a) SEM micrograph depicting a $\mathrm{Pt}$ alignment marker on the left, close to a growth defect. The dashed squares "1" and "2" indicate the positions of electrode deposition. (b) Current vs. timecurves of the two electrodes "1" and "2" (with and without defect, respectively).
The conducting substrate was contacted via Al-wire bonding, and electrical characterization was performed using an Agilent B 1500A semiconductor analyzer, with the voltage applied to the Pt top electrode and the substrate on ground potential. Figure 4(b) shows the log-log plots of current vs. time for an applied voltage of $+10 \mathrm{~V}$ for the two top electrode locations. The current measured at location " 2 " (unperturbed film) is initially very low and increases gradually over time, following the well-known degradation behavior for acceptor-doped $\mathrm{SrTiO}_{3} .{ }^{14}$ No forming was observed for over $30 \mathrm{~min}$ of applied voltage.

In contrast, the current measured at location " 1 " (on top of the defect) is initially almost four orders of magnitude larger, and reaches the forming current of $5 \mathrm{~mA}$ after just $1.8 \mathrm{~s}$, which is three orders of magnitude less than electrode "2." The cell can be switched into the low-resistance SET state using a negative bias at the top electrode, and into the high-resistance RESET state with a positive bias, as can be seen in Figure 5. This switching polarity is in agreement with the model of an oxygen-deficient filament that is created during forming and which is reversibly reduced and reoxidized near the top electrode during switching. ${ }^{1,8,15,16}$

It should be noted that the impact of such defects strongly affects any statistical analysis that does not explicitly take them into account. During an investigation that was conducted without explicitly checking for defects, a remarkable $13 \%$ out of 492 electrodes showed fast-forming as it can be observed for the case described above.

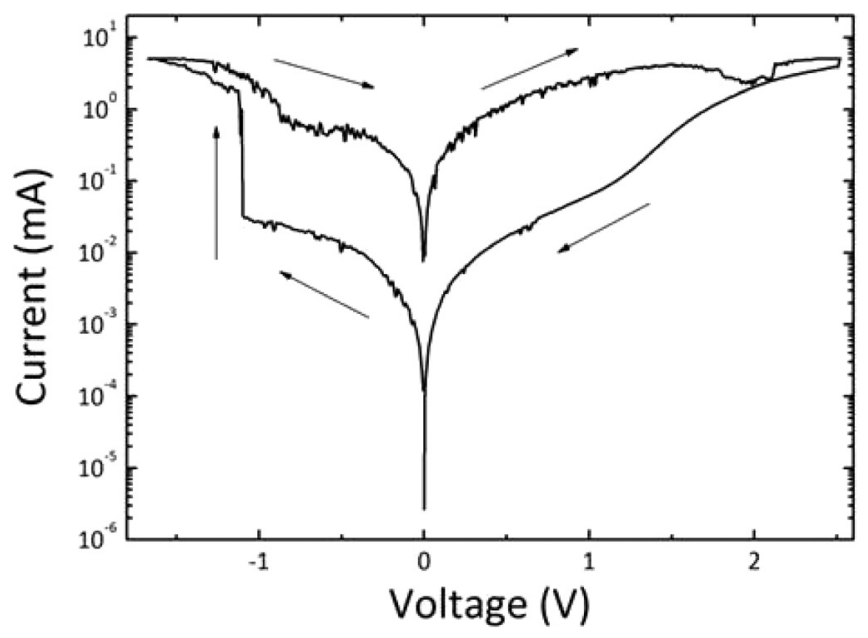

FIG. 5. Quasistatic current-voltage characteristics of the MIM cell containing the growth defect indicated in area "1" after electroforming is completed. The arrows indicate the sweep direction of the voltage.
The physical mechanism of the fast-forming behavior found at screw-dislocation exits is likely related to two issues: (i) the polycrystalline character of the film around the defect and (ii) the altered geometry caused by the faster growth. For the first issue, the key quality of the defect is the presence of grain boundaries. Grain boundaries in acceptordoped $\mathrm{SrTiO}_{3}$ contain high concentrations of oxygen vacancies $\mathrm{V}_{O}^{*}$, and therefore create a double Schottky-barrier between grains. ${ }^{17,18}$ While the ionic transport of oxygen vacancies is electrostatically hindered perpendicular to the grain boundaries, the higher electron concentrations and large concentration of defects define the grain boundaries as electrically conducting pathways for current flowing along the grain boundaries. For nanocrystalline $\mathrm{SrTiO}_{3}$, the electrical conduction has been found to derive exclusively from the grain boundaries due to the overlapping space charge zones. ${ }^{19}$ The high current density along the grain boundaries leads to the observed large initial current, which in turn induces local Joule heating that can accelerate ionic transport in the system and lead to the fast-forming. ${ }^{3}$

For the latter, the geometrical alteration caused by the fast-growing defect (compare Figure 3) leads to sharp edges in the top electrode layer where the metal covers the elevated part of the defect, which creates high local electric fields at the edges. These edges can lead to localized electrical currents as was previously observed in Ref. 13, which reinforces the Joule heating effect.

In summary, we have shown that the presence of screw dislocation exits in the substrate surface has a great impact on the electrical properties of MIM structures fabricated from resistively switchable Fe-doped $\mathrm{SrTiO}_{3}$. The drastic changes in growth environment close to the dislocations result in polycrystalline defects that act as fast-forming sites due to their higher electronic conductivity and increased oxygen diffusion through local Joule heating. These findings highlight the demand for extraordinary control over the defect structure of oxide materials for future memory applications.

This work was funded in part by the Deutsche Forschungsgemeinschaft (SFB 917) and in part by the EC FP7 MATERA project "Functional materials for resistive switching memories" (FMRSM) and the NRW-EU Ziel 2-Program. The authors gratefully acknowledge the support of Mr. Connell by the National Science Foundation and the NNIN.

${ }^{1}$ R. Waser, R. Dittmann, G. Staikov, and K. Szot, Adv. Mater. 21, 2632 (2009).

${ }^{2}$ A. Sawa, Mater. Today 11, 28 (2008). 
${ }^{3}$ S. Menzel, M. Waters, A. Marchewka, U. Böttger, R. Dittmann, and R. Waser, Adv. Funct. Mater. 21, 4487 (2011).

${ }^{4}$ M. Sowinska, T. Bertaud, D. Walczyk, S. Thiess, M. Schubert, M. Lukosius, W. Drube, C. Walczyk, and T. Schroeder, Appl. Phys. Lett. 100, 233509 (2012).

${ }^{5}$ J. P. Strachan, D. B. Strukov, J. Borghetti, J. J. Yang, G. MedeirosRibeiro, and R. S. Williams, Nanotechnology 22, 254015 (2011).

${ }^{6}$ T. Menke, P. Meuffels, R. Dittmann, K. Szot, and R. Waser, J. Appl. Phys. 105, 066104 (2009).

${ }^{7}$ J. P. Strachan, G. Medeiros-Ribeiro, J. J. Yang, M.-X. Zhang, F. Miao, I. Goldfarb, M. Holt, V. Rose, and R. S. Williams, Appl. Phys. Lett. 98, 242114 (2011).

${ }^{8}$ Ch. Lenser, A. Kuzmin, J. Purans, A. Kalinko, R. Waser, and R. Dittmann, J. Appl. Phys. 111, 076101 (2012).

${ }^{9}$ S. Stille, Ch. Lenser, R. Dittmann, A. Koehl, I. Krug, R. Muenstermann, J. Perlich, C. Schneider, U. Klemradt, and R. Waser, Appl. Phys. Lett. 100, 223503 (2012).
${ }^{10}$ R. Waser and M. Aono, Nature Mater. 6, 833 (2007).

${ }^{11}$ K. Szot, R. Dittmann, W. Speier, and R. Waser, Phys. Status Solidi (RRL) 1, R86 (2007).

${ }^{12}$ Z. Zhang, W. Sigle, and W. Kurtz, Phys. Rev. B 69, 144103 (2004).

${ }^{13}$ C. Rossel, G. I. Meijer, D. Brémaud, and D. Widmer, J. Appl. Phys. 90, 2892 (2001).

${ }^{14}$ R. Waser, T. Baiatu, and K. H. Hardtl, J. Am. Ceram. Soc. 73, 1645 (1990).

${ }^{15}$ T. Menke, R. Dittmann, P. Meuffels, K. Szot, and R. Waser, J. Appl. Phys. 106, 114507-1 (2009).

${ }^{16}$ M. Janousch, G. I. Meijer, U. Staub, B. Delley, S. F. Karg, and B. P. Andreasson, Adv. Mater. 19, 2232 (2007).

${ }^{17}$ S. Rodewald, J. Fleig, and J. Maier, J. Am. Cream. Soc. 84, 521 (2001).

${ }^{18}$ R. de Souza, J. Fleig, J. Maier, Z. Zhang, W. Sigle, and M. Ruehle, J. Appl. Phys. 97, 053502 (2005).

${ }^{19}$ P. Balaya, M. Ahrens, L. Kienle, J. Maier, B. Rahmati, S. B. Lee, W. Sigle, A. Pashkin, C. Kuntscher, and M. Dressel, J. Am. Ceram. Soc. 89, 2804 (2006). 